\title{
Observation of Stress Wave and Thermal Stress in Ultrashort Pulse Laser Bulk Processing inside Glass
}

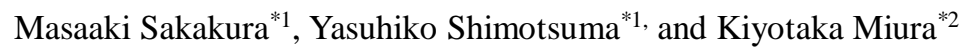 \\ ${ }^{* 1}$ Next Generation Laser Processing Technology Research Association, Kyoto 615-8245, Japan \\ E-mail: sakakura@laserprocessing.jp \\ *2 Dept. of Material Chemistry, Graduate School of Eng., Kyoto Univ., Kyoto, 615-8510, Japan
}

\begin{abstract}
Stress dynamics, stress wave and thermal stress, after photoexcitation inside glass a by tightly focused ultrashort laser pulse were investigated by a pump-probe polarization microscope and Transient Lens (TrL) method. The observation by the pump-probe polarization microscope revealed transient birefringence distributions of a laser-induced stress wave and thermal stress. The directions of compressive stress by a stress wave and thermal stress were radial from the photoexcitation, and the amplitudes of the stress estimated from the retardance of the birefringence were as high as several hundreds MPa. The observation by the TrL measurement showed the propagation of a laser induced stress wave as an oscillating TrL signals. From the pulse duration dependence of the oscillation amplitudes of the TrL signals, we found that the threshold and sensitivity to pulse energy of the stress wave become higher at longer pulse duration. We interpreted the pulse duration dependence in terms of different ionization mechanism; multiphoton ionization should be dominant for shorter pulse durations, while avalanche ionization should be dominant for longer pulse duration. DOI: $10.2961 /$ jlmn.2017.02.0019
\end{abstract}

Keywords: femtosecond laser processing, pump-probe, dynamics, glass, stress wave, ionization process

\section{Introduction}

Ultrashort pulse (USP) laser is an indispensable tool for fabricating various microscale three dimensional structures, such as optical waveguides and microchannels, inside transparent materials [1-4]. In USP laser processing, localized modifications can be induced by inducing photoexcitation only in the laser focal region with a tightly focused laser pulse. The photoexcitated region should have experienced large stress, such as the generation of a strong stress wave, because the laser-matter interaction time is much shorter than the elastic relaxation time [5-13]. Strong stress waves influence on structural changes in and around the photoexcited region, for example, densification [1], crack generation [6] and dislocations [14]. In addition, the temperature in the photoexcited region should have been high enough to generate large thermal stress even after the laser induced stress wave propagated away. Because the thermal diffusion time is as long as several hundreds of nanoseconds, thermal stress can influence on the photoexcited region in a much longer time than a stress wave $[5,13,15]$. Therefore, stress dynamics around the photoexcited region from picoseconds to microseconds time range is important for elucidating the mechanism of material modification by irradiation with a focused USP laser pulse.

The authors have investigated the stress and thermal dynamics in the time range from picoseconds to microseconds using two kinds of pump-probe techniques; one is a pump-probe polarization microscope [6], and the other is Transient Lens (TrL) method [7-9]. A pump-probe polarization microscope observes the photoelastic effect after photoexcitation. Because this method can measure the birefringence distribution originated from stress in transparent ma- terials, the stress amplitude can be estimated from the measurement. On the other hand, a TrL method observes diffraction patterns of the probe light originated from the spatial modulation of the refractive index inside a material after photoexcitation. Because this method is sensitive to small refractive index change by a small stress wave, the pulse energy and pulse duration dependences of a stress wave can be investigated in detail. By combination of these two methods, we can reveal various aspects of the physics in USP laser processing.

In this paper, we reviewed our studies on stress dynamics in USP laser processing by two time-resolved observation techniques. These observations will provide us with insight on the mechanism of laser-induced modification in solid materials as well as the advantage of USP lasers in precise material processing. First, we showed the observation of laser-induced pressure wave and thermal diffusion inside glass by a pump-probe transmission microscope and polarization microscope. Based on the observed stress dynamics, we estimated the stress amplitude at a stress wave. Next, we showed that a stress wave can be observed under lower excitation energy by a TrL method. Based on the measurement of the relative amplitudes of a stress wave by a TrL method, we discussed the influence of pulse duration on photoexcitation and the advantages of using femtosecond laser over picosecond laser in precise laser processing.

\section{Experiments}

\subsection{Laser sources and samples}

The source of the pump pulses was mode-locked Tisapphire laser with a regenerative amplifier (Mira-Legend; Coherent Inc.). The central wavelength of the laser pulse 
was $800 \mathrm{~nm}$, the pulse duration was tunable from 100 fs to $2 \mathrm{ps}$, and the repetition rate of the output pulses was $30 \mathrm{~Hz}$. The fundamental pulse $(\lambda=800 \mathrm{~nm})$ was used for a pump pulse. For detection of the dynamics after a single photoexcitation, a single pulse was extract using a mechanical shutter. The pulse energy was controlled by a neutral density filter.

Different probe pulses were used for shorter $(<20 \mathrm{~ns})$ and longer ( $>20 \mathrm{~ns}$ ) time ranges. For the shorter time range, the probe pulse was the second harmonics $(\lambda=400 \mathrm{~nm})$, which was obtained by passing the fundamental pulse through a BBO crystal. For the longer time range (after 20 ns) in a pump-probe polarization microscope, the probe pulse was a laser pulse of $1 \mu$ s pulse width which was generated from a laser diode ( $\lambda=405 \mathrm{~nm})$ and the probe light was detected by a CMOS camera with an imaging intensifier (Andor Technology; iStar sCMOS). The time resolution in the longer time range was determined by the gate time of the imaging intensifier. The times of the probe pulse and the camera in the longer time were controlled by a trigger pulse through a digital delay generator (Stanford Research Systems; DG535) from the trigger pulse of the USP laser source.

The sample was a sodalime glass plate (SCHOTT; B270 Superwite) and silica glass plate (ShinEtsu QUARTS; VIOSIL-SQ). Typically, the size of the glass plates was 75 $\mathrm{mm}$ in width, $25 \mathrm{~mm}$ in height and $1.0 \mathrm{~mm}$ in thickness. Pump pulses were incident normal to the largest surface of the glass plate. In pump-probe microscope measurements, the glass plate was translated normal to the laser propagation direction 0.2 seconds after a single pump-probe measurement. This is necessary for the observation of the dynamics in the region where there was no influence by the previous laser-induced modification. On the other hand, in TrL measurements, the glass plate was translated at about $10 \mathrm{~mm} / \mathrm{s}$ continuously during the measurement to avoid multiple photoexcitation at the same region. The translation velocity is short enough not to affect the observation of the dynamics in several nanoseconds, because the movement of the sample at $10 \mathrm{~mm} / \mathrm{s}$ in $10 \mathrm{~ns}$ is as short as $0.1 \mathrm{~nm}$.

(a)

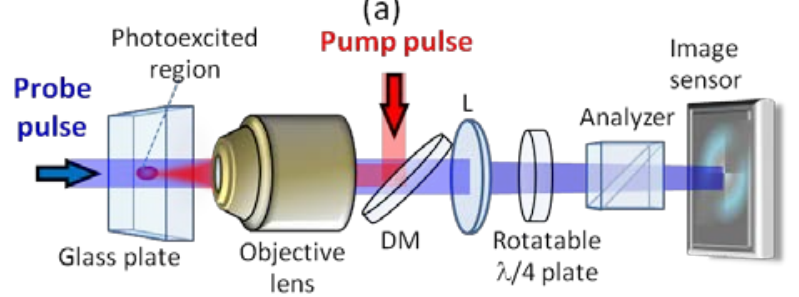

(b)

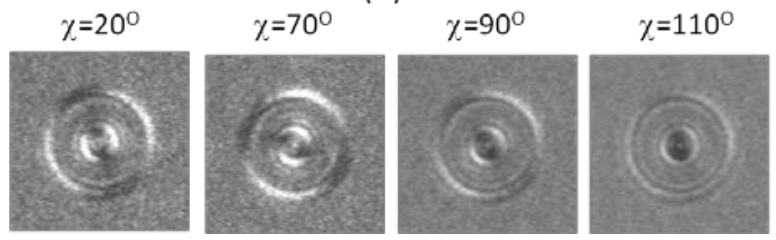

Fig. 1. (a) Optical setup of a pump-probe transmission and polarization microscope. L: Lens; DM: Dichroic mirror; $\lambda / 4$ plate: quarter waveplate. (b) Light intensity images captured at the different orientations of the $\lambda / 4$ plate $(\chi)$.

\subsection{Pump-probe transmission and polarization micro- scope}

Figure 1 shows an optical setup for pump-probe microscope. A pump pulse with a diameter of about $5 \mathrm{~mm}$ was focused inside a glass plate with a $50 \times$ objective lens $(f=4$ $\mathrm{mm}, \mathrm{NA}=0.8$ ) to induce photoexcitation at the focus. The position of the focus was about $0.1 \mathrm{~mm}$ from the glass surface. At the same time, an optically delayed probe pulse with a diameter of about $4 \mathrm{~mm}$ was transmitted through the photoexcited region from the opposite direction of the pump pulse. The transmitted probe pulse was expanded by the same objective lens, and the image of the photoexcited region was focused on an imaging sensor. After a single pump-probe measurement, the glass plate was translated normal to the laser propagation direction for the next pump-probe to measure the dynamics in the region where there was no influence by the previous laser-induced modification.

For transmission imaging, the intensity profile of the probe pulse was detected without the polarizer before the imaging sensor. For polarization imaging, the intensity profile was measured with the polarizer, and several intensity profiles were measured with a waveplate of different orientations $(\chi)$. Figure 1(b) shows different transmission images measured at different orientations of the waveplates. By analysis of the different transmission images as a function of $\chi$, the distribution of birefringence in the sample was obtained. The analysis for obtaining the birefringence is described in our previous paper in detail [6].

(a)

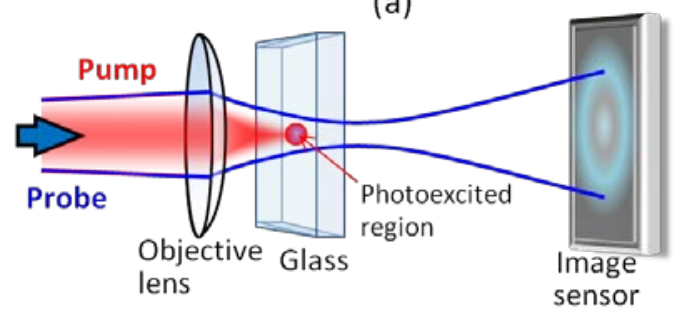

(b)

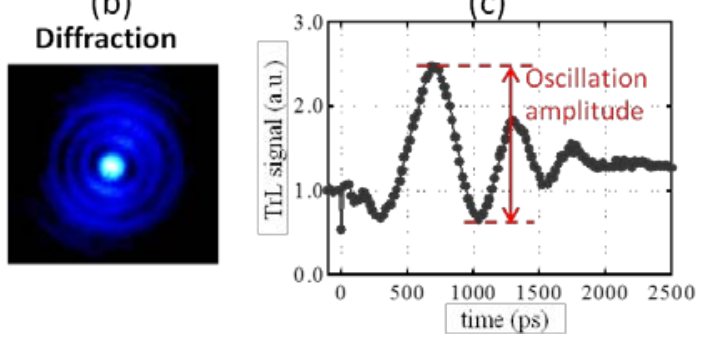

Fig. 2. (a) Schematic illustration of a TrL method for fs laser bulk processing inside a glass. (b) An example of a diffraction pattern obtained by the TrL measurement. The delay time was 600 ps after the photoexcitation. (c) An example of a TrL signal of a stress wave inside a glass.

\subsection{Transient Lens (TrL) method}

Figure 2(a) shows a simplified picture of a TrL measurement for laser bulk processing inside a glass. A pump pulse with a diameter of about $5 \mathrm{~mm}$ was focused inside a glass with a $50 \times$ objective lens $(f=10 \mathrm{~mm}, \mathrm{NA}=0.45)$ to induce nonlinear ionization only in the focal region. The focal position was about $0.2 \mathrm{~mm}$ from the glass surface. At the same time, an optically delayed probe pulse with a di- 
ameter of about $4 \mathrm{~mm}$ was passed along the same path as the pump pulse and transmitted through the photoexcited region. The focus of the probe pulse was about $0.06 \mathrm{~mm}$ apart from that of the pump pulse so that the diffraction pattern appears clearly at the far field. The diffraction pattern was imaged on an imaging sensor as a TrL image. The diffraction pattern is shown in Fig. 2 (b). The circularly symmetric diffraction pattern was due to the generation of cylindrically-shaped stress wave from the photoexcited region. In a similar way to the conventional TrL methods, the intensities at the central region of the diffraction patterns were measured as a function of the time delays as a "TrL signal". The intensities of a TrL signal were normalized by the signal intensity before the photoexcitation. Figure 2 (c) shows a TrL signal after fs laser irradiation inside a sodalime glass. As described in our previous study [7], the oscillating TrL signal can be observed when a stress wave was generated inside a glass. Because a stronger stress wave generates a TrL signal of larger oscillation, the amplitude of a stress wave can be estimated from the amplitude of the TrL oscillation [7-9]. In this study, the oscillation amplitude was measured as the difference between the signal intensity at the first positive peak and that at the second negative peak.

Another interesting point in the TrL signal in Fig. 2 (c) is the quasi-static signal intensity after the decay of the oscillation. The quasi-static signal intensity reflects the refractive index distribution after a laser-induced stress wave propagated away from the observed region. The refractive index distribution should be attributed to temperature and density distributions. In the measurement condition in this study (the probe beam was focused prior to the probe pulse), a concave lens-shaped refractive index distribution (refractive index decrease in the photoexcited region) results in higher quasi-static signal intensity. However, it is difficult to estimate the complicated refractive index distribution, for example, combination of multiple concave and convex lens, only from the signal intensity. Therefore, we will not discuss the quasi-static signal intensity in this study. The quasi-static TrL signal has been reported in our previous paper [7].

\section{Results}

\subsection{Stress wave generation inside glass}

Figure 3 (a) shows the transient transmission microscope images at different time delays after focusing $100 \mathrm{fs}$ laser pulses of $2 \mu \mathrm{J}$ inside a sodalime glass. The photoexcited region in the central region of the image became dark after the photoexcitation, and a circular wave propagated away from the photoexcited region. Because the velocity of the circular wave is comparable to the sound velocity in the glass $(\sim 6 \mu \mathrm{m} / \mathrm{ns})[8,10]$, this wave should be attributed to a laser-induced stress wave.

The birefringence distributions measured at the same time delays are shown in Figs. 3 (b) and 3 (c). In Fig. 3 (c), the slow axis of the birefringence was expressed by colors; for example red color means $0^{\circ}$ and yellow color means about $160^{\circ}$. The birefringence distributions around the photoexcited region and at the stress wave were observed much more clearly than the transmission images. The slow axis of the birefringence was radial from the photoexcited region both around the photoexcited region and in the stress wave. The distribution of the slow axis suggests that the compressive stress was generated from the photoexcited region to the radial direction by the expansion in the photoexcited region, such as thermal expansion. (a)
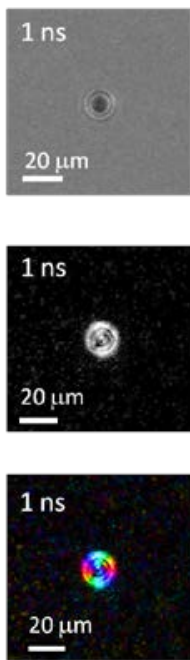
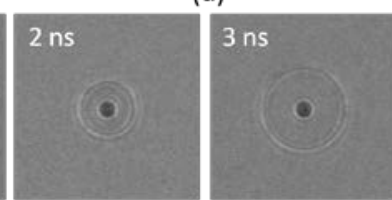

(b)
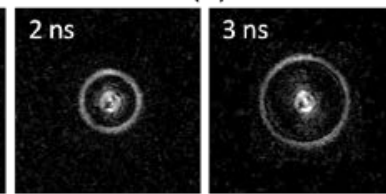

(c)
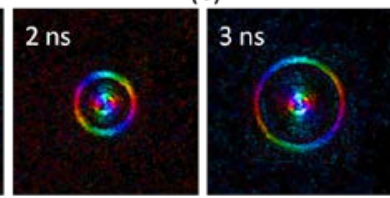
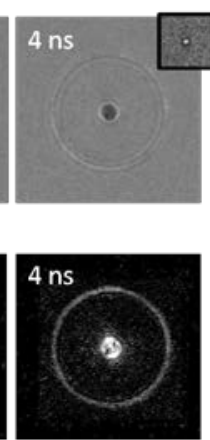
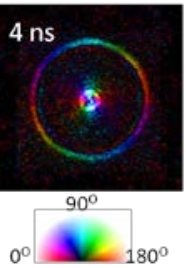

Fig. 3. Observed images at different time delays after fs laser irradiation inside a sodalime glass. (a) Transmission images. The inset shows the transmission image of the completely relaxed modification. (b), (c) Distributions of birefringence. (b) for retardance, and (c) for slow axis of the birefringence. The relation between slow axis and color is given by the colored semicircle. (a)

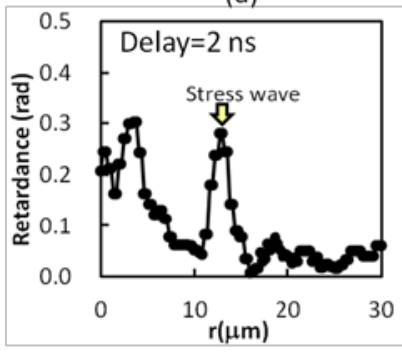

(b)

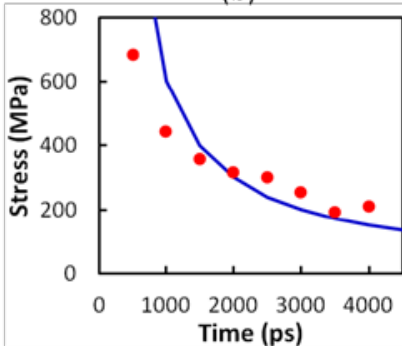

Fig. 4. (a) The distribution of the retardance plotted against the radial position $(r)$ from the center of the photoexcited region. The retardance band by a stress wave is around $r=12$ $\mu \mathrm{m}$. (b) The calculated stresses plotted agains time delays. Red closed circules are the calculated stresses and the blue line is a curve expressed by $\sigma=\mathrm{A} t^{-1}$.

Because the observed birefringence should have been the result of photoelastic effect that is induced by strain and stress in a material, transient stress can be estimated using the relation between stress $(\sigma)$ and induced retardance $(\delta)$ [16]:

$$
\delta=2 \pi \frac{n l}{\lambda} C_{\lambda} \sigma
$$

where $n$ is the refractive index, $l$ is the light propagation length in the stressed region, $\mathrm{C}_{\lambda}$ is the stress-optical coefficient and $\lambda$ is the wavelength of the probe light. The peak stress amplitude in the stress wave was estimated using Eq. (1), the reported values of a sodalime glass, $n=1.5$ and 
$\mathrm{C}_{\lambda}=2.83 \mathrm{TPa}^{-1}$ [16] and $\lambda=0.4 \mu \mathrm{m}$. Figure 4(a) shows the radial distribution of the observed retardance at 2 ns after the photoexcitation. The retardance at the peak in the stress wave $(r=12 \mu \mathrm{m})$ was $\delta=0.3$. For the estimation of the stress, we assumed the length of the stressed region, $l$, was comparable to the length of the modification in the laser propagation direction, which was measured from the optical microscope, $l=20 \mu \mathrm{m}$. As the result, the stress in the stress wave at the peak was calculated to be $\sigma=225 \mathrm{MPa}$. In a similar manner, the peak stress in the stress wave were calculated for all the time delays and plotted against the time delay (Fig. 4(b)). The decay of the stress followed the inverse of the distance $(1 / r)$ after 1.5 ns approximately (the blue line in Fig. 4 (a)). The decay indicates that the photoexcited region was not a point-like but a line-shaped, because the stress wave from a point-like follows $1 / r^{1.5}$, which decays much faster. However, the stress in 1 ns deviated from the $1 / r$ curve. This deviation could be attributed to that the stress growth [10] had occurred in 1 ns in this laser irradiation condition.

(a)

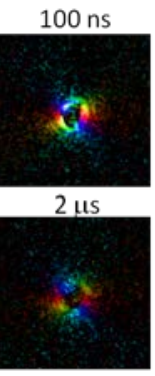

$-10 \mu \mathrm{m}$
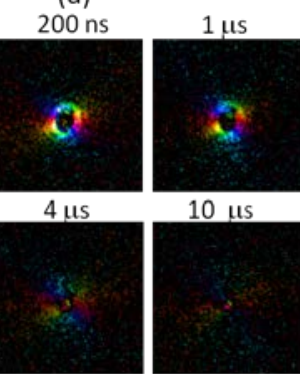

Fig. 5.. (a) Birefringence distributions in a longer time range after photoexcitation inside a sodalime glass with a focused fs laser pulse. The pulse enegy was $2 \mu \mathrm{J}$. (b) The maximum retardance in the observed birefringence distribution plotted against the time after the photoexcitation.

\subsection{Thermal stress and diffusion observed by a polari- zation microscope}

Birefringence around the photoexcited region was still observed even in a longer time range after the stress wave propagated away from the observed region,. Figure 5 (a) shows the temporal change of the distributions of birefringence. The ring-shaped birefringence distribution around the photoexcited region decayed in several microseconds. The birefringence should be attributed to the thermal stress due to thermal expansion in the photoexcited region, because the decay time can be explained by the thermal diffusion inside the glass [9]. The thermal diffusion time $\tau_{\text {th }}$ can be estimated roughly by [17]

$$
\tau_{\text {th }}=\frac{d^{2}}{4 D_{t h}}
$$

where $d$ is the diameter of the initially heated region (i.e, photoexcited region) and $D_{t h}$ is the diffusion coefficient of the glass. Using $D_{t h}=0.45 \mu \mathrm{m}^{2} \cdot \mu$ s of sodalime glass [18] and $d=2 \mu \mathrm{m}$, the roughly estimated diameter of the photoexcited region, the thermal diffusion time is calculated as $\tau_{\text {th }}=2.2 \mu$ s. Figure 5 (b) shows the maximum birefringence measured against the delay time. The decay time was about
$2 \mu$ s, which is consistent to the thermal diffusion time estimated by Eq. (2).

The ring-shaped birefringence distribution suggests that thermal expansion had occurred in the photoexcited region and the thermal stress had compressed the surrounding solid region. In the similar manner as the previous section, the thermal stress was estimated by the observed birefringence and Eq. (1). Using the same values as the calculation in the previous section $\left(n=1.5\right.$ and $\mathrm{C}_{\lambda}=2.83 \mathrm{TPa}^{-1}, \lambda=0.4$ $\mu \mathrm{m}$ and $l=20 \mu \mathrm{m})$ and $\delta=0.58$ obtained from the birefringence distribution at $100 \mathrm{~ns}$, the thermal stress, $\sigma_{\text {th }}$, at 100 ns was estimated as $\sigma_{\mathrm{th}}=435 \mathrm{MPa}$. The relation between the thermal stress and temperature change $(\Delta T)$ is expressed by

$$
\sigma_{t h}=\frac{E}{3(1-2 v)} \beta \Delta T
$$

where $E, v$ and $\beta$ are the Young modulus, Poisson ratio, and thermal expansion coefficient of sodalime glass, respectively. Using the estimated stress $\sigma_{\mathrm{th}}=435 \mathrm{MPa}, E=72 \mathrm{GPa}$, $v=0.22$ and $\beta \sim 9 \times 10^{-6} \mathrm{~K}^{-1}$ of sodalime glass [19], the temperature change can be calculated by Eq. (3) as $\Delta \mathrm{T}=1100 \mathrm{~K}$ This temperature change is underestimated, because the estimated point is several $\mu \mathrm{m}$ apart from the center. Notwithstanding the underestimation, the calculated temperature change suggests that the glass in the photoexcited region had been heated much above the softening temperature $\left(\sim 720^{\circ} \mathrm{C}\right)$ in several hundreds of nanoseconds.

\subsection{Amplitude of a stress wave by a $\operatorname{TrL}$ method}

In the section 3.1, the stress wave propagation inside a sodalime glass after focusing a 100 fs laser pulse were shown as transmission images and birefringence distribution. Although a pump-probe polarization microscope can visualize the shape of the stress wave clearly, the method cannot detect much weaker stress wave which is generated by much smaller laser energy. On the other hand, a TrL method can detect a small stress wave, because a propagating stress wave generates a clear oscillating TrL signal as shown in Fig. 2(c). The mechanism of oscillating signal by a propagating wave has been explained in our previous paper in detail [6]. In this study, we investigated the pulse energy dependence of a stress wave by the measurement of oscillating TrL signals and compared the thresholds and sensitivity of stress wave generation by different pulse durations.
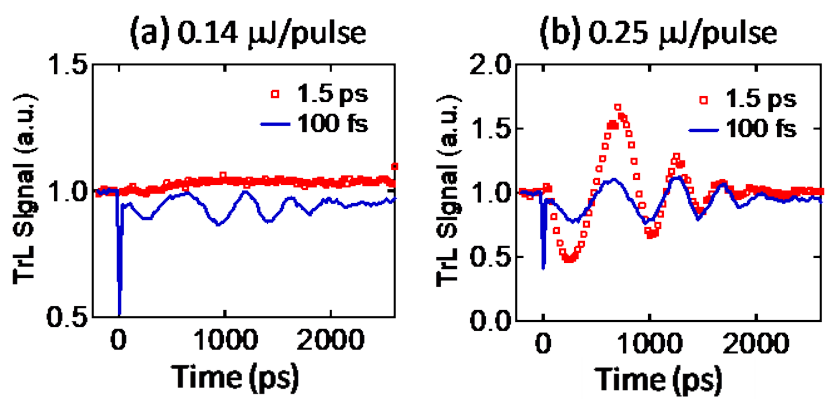

Fig. 6. TrL signals after photoexcitation by focused laser pulses of different pulse durations. The pulse energy was (a) $0.14 \mu \mathrm{J}$ and (b) $0.25 \mu \mathrm{J}$. The decrease of the signal intensity at 0 ps was caused by a refractive index change due to an optical Kerr effect. 
Figure 6 shows the TrL signals after photoexcitation inside a silica glass by focused laser pulses of different pulse durations and different pulse energies. When the pulse energy was $0.14 \mu \mathrm{J}$ (Fig. 6 (a)), the oscillation of the TrL signal was clearly observed by a 100 fs laser pulse, while not by a 1.5 ps laser pulse. This difference suggests that the threshold of stress wave generation is lower at shorter pulse duration. On the other hand, when the pulse energy was $0.25 \mu \mathrm{J}$ (Fig. 6 (b)), the oscillation amplitude by a $1.5 \mathrm{ps}$ laser pulse became larger than that by a $100 \mathrm{fs}$ laser pulse. In the other words, the oscillation amplitude by a $1.5 \mathrm{ps}$ increased more steeply with increasing pulse energy.

The oscillation amplitudes measured at different pulse durations were plotted against the pulse energies in Fig. 7(a). Clearly, the oscillation appeared at a lower pulse energy at shorter pulse duration. On the other hand, the variation of the oscillation amplitude to pulse energy change is more steep at longer pulse duration. Figure 7 (b) and 7 (c) show the threshold of stress wave generation and the sensitivity to pulse energy change (the variation of the amplitude to pulse energy) as a function of the pulse duration, respectively. In these plot, the negative values of the pulse duration mean the sign of the chirp of the laser pulse. The variation of TrL signal to pulse energy change depended on the pulse duration, but the difference by the sign of the chirp was negligible. The negligible difference by the sign of the chirp suggests that the band width of the pump pulse ( $~ 8 \mathrm{~nm})$ was so small that the sign of the chirp could not affect the photoexcitation process inside a silica glass. The plot in Fig. 7 (b) shows that the threshold becomes higher with increasing pulse duration, and the plot in Fig. 7 (c) shows that a stress wave becomes stronger more steeply with increasing pulse energy at longer pulse duration.

Because the origin of a laser-induced stress wave is the stress in the photoexcited region [5, 8-10, 12], the pulse duration dependence of the stress wave suggests that the thermal energy by excited electrons should depend on the pulse duration. Therefore, we interpreted the pulse duration dependence of Fig. 7 according to the dynamics of photoexcited electrons. The simple rate equation of free electrons in the excited state in a laser field can be expressed by $[5,20,21]$

$$
\frac{d \rho}{d t}=\left(\frac{d \rho}{d t}\right)_{m p}+\eta_{\text {aval }} \rho-g \rho-\eta_{\text {rec }} \rho^{2}
$$

where $\rho$ is the free electron density in the excited state, $(\mathrm{d} \rho / \mathrm{d} t)_{m p}$ is the multiphoton absorption rate, $\left(\eta_{\mathrm{aval}} \rho\right)$ is the avalanche (or cascade) ionization rate, $(-g \rho)$ is the diffusion of free electrons from the photoexcited region, and $\left(-\eta_{\text {rec }} \rho^{2}\right)$ is the electron-hole recombination.

When $k$-photons are necessary for excitation of electrons in the material, the multiphoton ionization rate is roughly proportional to the $k$ th power of the laser light intensity (I) $[20,22]$, i.e. $(\mathrm{d} \rho / \mathrm{d} t)_{\mathrm{mp}} \propto I^{\mathrm{k}}$. Therefore, at the same pulse energy, the multiphoton absorption occurs more efficiently by a shorter pulse duration, because a shorter laser pulse has larger intensity. On the other hand, the avalanche ionization becomes dominant as the light-electron interaction time becomes longer [20,23]. The avalanche ionization induce exponential growth of free electrons, which is expressed by $\rho(t)=\rho_{0} \exp \left(\eta_{\text {aval }} t\right)$, the solution of Eq. (4) with the other contributions neglected [24,25]. (a)
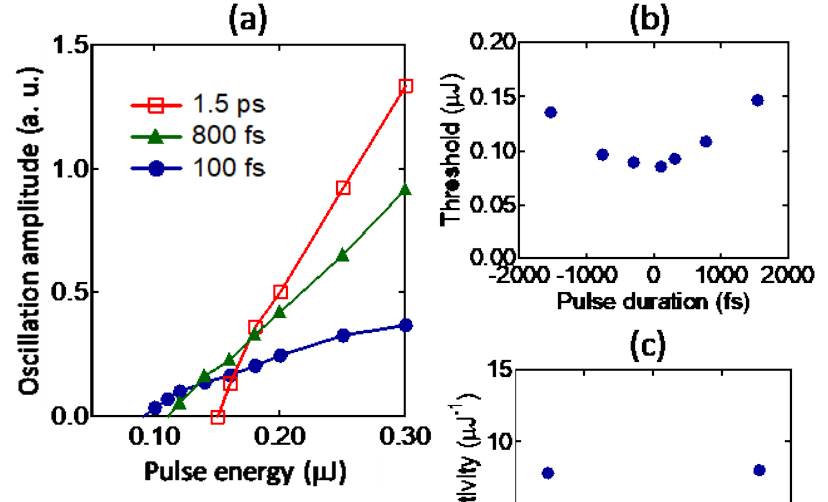

(c)

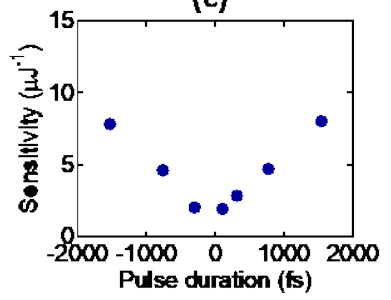

Fig. 7. (a) Oscillation amplitudes of TrL signals plotted against pulse energies for different pulse durations. (b) Threshold of stress wave generation for different pulse durations. (c) Sensitivity of an oscillation amplitude, which is the variation of oscillation amplitude to pulse energy change, for different pulse energy. In (b) and (c), the negative pulse duration means the negative chirp of the laser pulse.

The avalanche ionization accounts for the sensitivity to pulse energy change (Fig. 7(c)), because exponential growth of free electrons by avalanche ionization generates more thermal energy at a longer pulse duration. However, the avalanche ionization needs the seed electrons, $\rho_{0}$, which must be generated by multiphoton ionization. This means that a stress wave cannot be generated when the multiphoton ionization is too small to generate numbers of seed electrons enough to induce avalanche ionization. Considering that the multiphoton ionization rate, $(\mathrm{d} \rho / \mathrm{d} t)_{m p}$, is proportional to the $k$-th power of laser intensity [20,22], a shorter laser pulse can generate enough numbers of seed electrons at lower pulse energy. Therefore, the lower threshold of a stress wave at shorter pulse duration should be attributed to the initial excitation of electrons by multiple ionization.

Finally, we give some suggestions for the choice of laser from the pulse duration dependence of a stress wave found by the TrL measurement. The first suggestion is for the application of ultrashort pulsed laser to stress wave generation; if we need to generate stronger stress waves, we should use picosecond lasers. This suggestion comes from the observation of a stronger stress wave by a 1.5 ps laser pulse of $0.3 \mu \mathrm{J}$ in Fig. 7 (a), of which amplitude was about three times larger than that by a 100 fs pulse. The second suggestion is for the application of ultrashort pulsed laser to precise laser processing; if we need to control laserinduced modifications precisely, we should use femtosecond laser. This suggestion comes from the lower sensitivity of the amplitude of a stress wave to pulse energy variation at shorter pulse duration (Fig. 7 (c)). Owing to the lower sensitivity, modifications by shorter laser pulses would not be influenced by the fluctuation of pulse energies, which could come from dusts on the sample surface and defects in the sample. The robustness to the fluctuation of pulse energies enables precise control of laser-induced modification. 


\section{Summary and Conclusion}

The observations of laser-induced stress waves by pump-probe polarization microscope and TrL method are reviewed. The observation by the pump-probe microscope visualized the generation, propagation and decay of a laser induced stress wave. The stress amplitude was estimated by the observed birefringence at the stress wave. The observation by the TrL method elucidated the pulse duration dependence of the laser induced stress wave. Both the threshold of the stress wave generation and the sensitivity of the amplitude to pulse energy variation were higher at longer pulse duration. The pulse duration dependences were interpreted by photoexcitation process of electrons in glass; for shorter pulse duration, multiphoton ionization is dominant, while for longer pulse duration, avalanche ionization becomes dominant. Finally, we gave two suggestions for laser application based on the pulse duration dependence; a longer pulsed laser is preferable for generation of stronger stress waves, while a shorter pulsed laser is preferable for precise laser processing.

\section{Acknowledgments}

The author, MS, appreciates Prof. Bulgakova and Prof. Stoian for fruitful discussion and valuable comments to the results by the TrL measurement. This work was supported by the Cross-Ministerial Strategic Innovation Promotion (SIP) Program, and JSPS KAKENHI grants No. 18760548, No. 22750187 and No. 26410240.

\section{References}

[1] K. Miura, J. R. Qiu, H. Inouye, T. Mitsuyu and K. Hirao: Appl. Phys. Lett., 71, (1997) 3329.

[2] L. Shah, A. Y. Arai, S. M. Eaton and P. R. Herman: Opt. Express, 13, (2005) 1999.

[3] H. B. Sun, Y. Xu, S. Juodkazis, K. Sun, M. Watanabe, S. Matsuo, H. Misawa and J. Nishii: Opt. Lett., 26, (2001) 325.

[4] K. Sugioka and Y. Cheng: Appl. Phys. Rev., 1, (2014) 041303.

[5] A. Vogel, J. Noack, G. Huttman and G. Paltauf: Appl. Phys. B, 81, (2005) 1015.

[6] M. Sakakura, Y. Shimotsuma, N. Fukuda and K. Miura: J. Appl. Phys., 118, (2015) 023106.

[7] M. Sakakura, M. Terazima, Y. Shimotsuma, K. Miura and K. Hirao: J. Appl. Phys., 109, (2011) 023503.

[8] M. Sakakura and M. Terazima: Phys. Rev. B, 71, (2004) 024113.

[9] M. Sakakura, M.Terazima, Y. Shimotsuma, K. Miura and K. Hirao: Laser Chemistry, 2010, (2010) 1.

[10] M. Sakakura, M. Terazima, Y. Shimotsuma, K. Miura and K Hirao: Opt. Express, 15, (2007) 5674.

[11] S. I. Kudryashov, V. D. Zvorykin, A. A.Ionin, V. Mizeikis, S. Juodkazis and H. Misawa: Appl. Phys. Lett., 92, (2008)101916.

[12] A. Mermillod-Blondin, J. Bonse, A. Rosenfeld, I. V. Hertel, $\square$ Yu. P. Meshcheryakov, N. M. Bulgakova, E. Audouard and R. Stoian: Appl. Phys. Lett., 94, (2009) 041911. [13] Y. Hayasaki, M. Isaka, A. Takita, S. Hasegawa and S. Juodkazis: Opt. Mat. Exp., 2, (2012) 691.

[14] S. Kanehira, K. Miura, K. Fujita, K. Hirao, J. Si, N. Shibata and Y. Ikuhara: Appl. Phys. Lett., 90, (2007) 163110 .
[15] M. Sakakura, M. Terazima, Y. Shimotsuma, K. Miura and K. Hirao: Opt. Exp., 115, (2007) 16800.

[16] A. K. Varshneya, "Fundamentals of Inorganic Glasses" (Academic Press, Boston, 1994) pp. 478-481.

[17] G. Paltauf and P. Dyer: Chem. Rev. 103, (2003) 487.

[18] M. Sakakura, M. Shimizu, Y. Shimotsuma , K. Miura and K. Hirao :Appl. Phys. Lett., 93, (2008) 231112.

[19] Glass data sheet from Schott:

http://psec.uchicago.edu/glass/Schott\%20B270\%20Propert ies\%20-\%20Knight\%20Optical.pdf

[20] J. Noack and A. Vogel: IEEE J. Quantum. Electron., 35, (1999) 1156.

[21] N. Bloembergen: IEEE J. Quantum Electron., QE-10, (1974) 375.

[22] C. B. Schaffer, A. Brodeur and E. Mazur: Meas. Sci. Tech., 12, (2001) 1784.

[23] B. C. Stuart, M. D. Feit, A. M. Rubenchik, B. W. Shore and M. D. Perry: Phys. Rev. Lett., 74, (1995) 2248.

[24] D. Du, X. Liu, G. Korn, J. Squier and G. Mourou: Appl. Phys. Lett., 64, (1994) 3071.

[25] M. H. Niemz: Appl. Phys. Lett., 66, (1995) 1181.

(Received: June 19, 2017, Accepted: September 10, 2017) 